\title{
Nonadministration of pharmacologic venous thromboembolism prophylaxis is less common in hospitalized patients with COVID-19
}

\author{
Mujan Varasteh Kia ${ }^{1}$ - Brandyn D. Lau ${ }^{2,3,4,11}$. Oluwafemi P. Owodunni ${ }^{1}$. Peggy S. Kraus ${ }^{9} \cdot$ Christine G. Holzmueller $^{4}$. \\ Deborah B. Hobson ${ }^{1,4,10} \cdot$ Dauryne L. Shaffer ${ }^{1,10} \cdot$ Michael B. Streiff ${ }^{4,5} \cdot$ Elliott R. Haut ${ }^{1,4,6,7,8,11,12}$ (1)
}

Accepted: 10 January 2021 / Published online: 28 January 2021

(C) The Author(s), under exclusive licence to Springer Science+Business Media, LLC part of Springer Nature 2021

\begin{abstract}
Introduction The incidence of venous thromboembolism (VTE) in patients hospitalized with COVID-19 is higher than most other hospitalized patients. Nonadministration of pharmacologic VTE prophylaxis is common and is associated with VTE events. Our objective was to determine whether nonadministration of pharmacologic VTE prophylaxis is more common in patients with COVID-19 versus other hospitalized patients.

Materials and methods In this retrospective cohort analysis of all adult patients discharged from the Johns hopkins hospital between Mar 1 and May 12, 2020, we compared demographic, clinical characteristics, VTE outcomes, prescription and administration of VTE prophylaxis between COVID-19 positive, negative, and not tested groups.

Results Patients tested positive for COVID-19 were significantly older, and more likely to be Hispanic, have a higher median body mass index, have longer hospital length of stay, require mechanical ventilation, develop pulmonary embolism and die (all $p<0.001$ ). COVID-19 patients were more likely to be prescribed (aOR 1.51, 95\% CI 1.38-1.66) and receive all doses of prescribed pharmacologic VTE prophylaxis (aOR 1.48, 95\% CI 1.36-1.62). The number of patients who missed at least one dose of VTE prophylaxis and developed VTE was similar between the three groups $(p=0.31)$.

Conclusions It is unlikely that high rates of VTE in COVID-19 are due to nonadministration of doses of pharmacologic prophylaxis. Hence, we should prioritize research into alternative approaches to optimizing VTE prevention in patients with COVID-19.
\end{abstract}

Keywords Venous thromboembolism · Deep vein thrombosis · Pulmonary embolism · Thromboprophylaxis · Missed doses $\cdot$ COVID-19

\section{Highlights}

- Nonadministration of pharmacologic venous thromboembolism (VTE) prophylaxis is common, but has not been studied in COVID-19.

- Administration of pharmacologic VTE prophylaxis was compared between COVID-19 positive, negative, and not tested groups.

Mujan Varasteh Kia, Brandyn D. Lau contributed equally to this work.

Elliott R. Haut

ehaut1@jhmi.edu

Extended author information available on the last page of the article
- Patients with COVID-19 were more frequently prescribed and administered pharmacologic VTE prophylaxis.

- After adjusting for confounders, hospitalized patients with COVID-19 had a similar chance of developing VTE compared to other patients.

\section{Introduction}

Patients with coronavirus disease 2019 (COVID-19) are at very high risk for hospital-associated venous thromboembolism (VTE) with reported event rates of 3.3 to $49 \%$ $[1,2]$. The prothrombotic phenotype of COVID-19 has been generally attributed to systemic inflammation [3]. We hypothesized that another potential cause might be nonadministration of VTE prophylaxis related to the unique 
challenges posed by COVID-19 care, including restrictions on direct patient contact and limited access to personal protective equipement (PPE). Pharmacologic VTE prophylaxis reduces the risk of preventable events by 30 to $65 \%$ [4]. In one study, VTE prophylaxis was associated with lower mortality in patients with severe COVID-19 infection and an elevated d-dimer [5]. Thromboprophylaxis, particularly low molecular weight heparin, is recommended therapy for hospitalized patients with COVID-19 unless contraindications are present [5]. We have reported frequent nonadministration of prescribed doses of VTE thromboprophylaxis among hospitalized patients $[6,7]$, which is associated with VTE $[8,9]$. Thus, we sought to determine whether nonadministration of pharmacologic VTE prophylaxis is more common in patients with COVID-19 compared to other hospitalized patients.

\section{Methods}

We retrospectively identified all adult patients discharged from The Johns hopkins hospital (JHH) between Mar 1 and May 12, 2020 (cut-off date reflects May 13 change to testing every admitted patient for COVID-19). Data were automatically extracted from the electronic medication administration record as in prior published studies [7, 10]. We compared demographics, clinical characteristics, VTE outcomes, and prescription and administration of VTE prophylaxis between three patient groups: COVID-19 positive, COVID-19 negative, and COVID-19 not tested. We analyzed missed doses of pharmacologic VTE prophylaxis and categorized documented reasons as patient refusal and other. The Chi-square test and Fisher's exact test compared categorical variables, and one-way analysis of variance (ANOVA) and quantile regression compared means and medians, respectively. To account for confounders, we calculated adjusted odds ratios (aORs) and 95\% confidence intervals (CI) using multiple logistic regression. Statistical significance was defined as $p<0.05$. The Johns hopkins medicine institutional review board approved this study.

\section{Results}

439 patients tested positive, 2316 tested negative, and 3035 were not tested for COVID-19. In comparing groups, the COVID-19 positive patient group was older and more likely to be Hispanic, had a higher body mass index and longer hospital length of stay, was more likely to require mechanical ventilation, and was more likely to die (Table 1).

Patients testing positive for COVID-19 were more often prescribed pharmacologic prophylaxis (87.2\%) compared to the COVID-19 negative (53.2\%) and not tested (49.3\%) patient groups $(p<0.001)$. Patients in the COVID-19 positive group missed significantly fewer prescribed doses (3.9\%) compared to the COVID-19 negative $(8.7 \%)$ and not tested $(8.0 \%)$ patient groups $(p<0.001)$. After adjusting for significant predictor variables, the COVID-19 positive group was more likely to be prescribed VTE prophylaxis (aOR $1.51,95 \%$ CI 1.38-1.66) and receive all prescribed doses

Table 1 Characteristics of patients discharged from the Johns hopkins hospital between Mar 1 and May 12, 2020

\begin{tabular}{|c|c|c|c|c|}
\hline Variable & $\begin{array}{l}\text { Patients tested }(+) \text { for } \\
\text { COVID-19 } \\
(\mathrm{n}=439)\end{array}$ & $\begin{array}{l}\text { Patients tested }(-) \text { for } \\
\text { COVID-19 } \\
(\mathrm{n}=2316)\end{array}$ & $\begin{array}{l}\text { Patients not tested } \\
(n=3035)\end{array}$ & $P^{\dagger}$ \\
\hline Age (SD), years & $55.5(17.9)$ & $48.3(21.0)$ & $47.7(23.6)$ & $<0.001$ \\
\hline Female, n (\%) & $218(49.7)$ & $1226(52.9)$ & $1589(52.4)$ & 0.65 \\
\hline Race, $\mathrm{n}(\%)$ & & & & $<0.001$ \\
\hline White & $115(26.2)$ & $1057(45.6)$ & $1508(49.7)$ & \\
\hline Black & $191(43.5)$ & $1051(45.4)$ & $1168(38.5)$ & \\
\hline Other $^{*}$ & $133(30.3)$ & $208(9.0)$ & $359(11.8)$ & \\
\hline Ethnicity, n(\%) & & & & $<0.001$ \\
\hline Hispanic or Latino & $86(19.6)$ & $101(4.4)$ & $159(5.2)$ & \\
\hline Median body mass index (IQR), $\mathrm{kg} / \mathrm{m}^{2}$ & $29.5(25.0,34.4)$ & $25.9(21.8,31.4)$ & $26.1(21.4,31.0)$ & $<0.001$ \\
\hline Median hospital length of stay (IQR), days & $7(4,12)$ & $4(2,7)$ & $4(2,6)$ & $<0.001$ \\
\hline Ventilated, n (\%) & $108(24.6)$ & $277(12.0)$ & $277(9.1)$ & $<0.001$ \\
\hline Death, n (\%) & $38(8.7)$ & $71(3.1)$ & $63(2.1)$ & $<0.001$ \\
\hline
\end{tabular}

$\mathrm{IQR}$, interquartile range; $\mathrm{SD}$, standard deviation

${ }^{\dagger}$ Mean age difference was compared using one-way analysis of variance (ANOVA); median body mass index and length of stay were compared between the three groups using quantile regression, and categorical variables were compared using the Chi-square test

* Other includes Asian, American Indian or Alaska Native, Native Hawaiian, other Pacific Islander, other, unknown/not reported, and declined to answer 
(aOR 1.48, 95\% CI 1.36-1.62). When examined at the dose level, doses were less likely to be missed (aOR $0.82 ; 95 \%$ CI $0.77-0.87$ ) or refused (aOR 0.76 ; 95\% CI 0.71-0.82) in the COVID-19 positive group.

On univariate analysis, the risk of developing VTE was significantly higher in patients with COVID-19, 11 (2.5\%) in patients who tested positive for COVID-19, 6 $(0.3 \%)$ in the COVID-19 negative group, and $15(0.5 \%)$ in the not tested group $(p<0.001)$. However, after adjusting for confounding factors, risk of developing VTE was similar between the three groups (aOR 1.08, 95\% CI 0.48-2.44). All patients with VTE in all three groups were prescribed VTE prophylaxis. Among patients with VTE, 36.4\% in the
COVID-19 positive group, 50\% in the COVID-19 negative group, and $66.6 \%$ in the not tested group missed at least one dose of prescribed prophylaxis $(p=0.31)$ (Table 2).

\section{Discussion}

We found that patients hospitalized with COVID-19 were more frequently prescribed and administered all doses of pharmacologic VTE prophylaxis compared to COVID-19 negative and non-tested patients. We suspect these findings reflect enhanced vigilance and prioritization by physicians (for prescription) and nurses (for administration) due to the

Table 2 Venous thromboembolism (VTE) events and nonadministration of pharmacologic VTE prophylaxis on both patient and dose levels comparing patients by COVID-19 testing Status (positive vs. negative vs. not-tested)

\begin{tabular}{|c|c|c|c|c|c|}
\hline & $\begin{array}{l}\text { Tested (+) for COVID- } \\
19\end{array}$ & $\begin{array}{l}\text { Tested (-) for COVID- } \\
19\end{array}$ & $\begin{array}{l}\text { Not tested for COVID- } \\
19\end{array}$ & $P^{\dagger}$ & $\begin{array}{l}\text { aOR }(95 \% \text { CI }) \text { Tested }(+) \\
\text { for COVID-19 versus } \\
\text { Others }\end{array}$ \\
\hline \multicolumn{6}{|l|}{ Patient visit level } \\
\hline $\begin{array}{l}\text { Total number of patient } \\
\text { visits }\end{array}$ & 439 & 2316 & 3035 & & \\
\hline $\begin{array}{l}\text { Prescribed pharmaco- } \\
\text { logic VTE prophy- } \\
\text { laxis, n }(\%)\end{array}$ & $383 / 439(87.2)$ & $1177 / 2136(53.2)$ & $1435 / 3035(49.3)$ & $<0.001$ & $1.51(1.38,1.66)^{\S}$ \\
\hline $\begin{array}{l}\text { Received all doses, } \mathrm{n} \\
(\%)\end{array}$ & $281 / 383(73.4)$ & $724 / 1177(61.5)$ & $917 / 1435(63.9)$ & $<0.001$ & $1.48(1.36,1.62)^{*}$ \\
\hline $\begin{array}{l}\text { Number of Patients } \\
\text { who Developed VTE } \\
\text { During Hospitaliza- } \\
\text { tion, n (\%) }\end{array}$ & $11 / 439(2.5)$ & $6 / 2136(0.3)$ & $15 / 3035(0.5)$ & $<0.001$ & $1.08(0.48,2.44)^{\S}$ \\
\hline DVT only & $4 / 439(0.9)$ & $1 / 2136(0.04)$ & $8 / 3035(0.3)$ & 0.005 & $0.92(0.41,2.07)^{\S}$ \\
\hline PE only & 6/439(1.4) & $5 / 2136(0.2)$ & $6 / 3035(0.2)$ & 0.002 & $1.93(0.94,3.95)^{\S}$ \\
\hline Both DVT and PE & $1 / 439(0.2)$ & $0 / 2136(0.0)$ & $1 / 3035(0.03)$ & 0.15 & $1.75(0.25,12.22)^{\S}$ \\
\hline $\begin{array}{l}\text { Patients with VTE who } \\
\text { were prescribed VTE } \\
\text { prophylaxis, n (\%) }\end{array}$ & 11/11 (100) & $6 / 6(100)$ & $15 / 15(100)$ & 1.00 & \\
\hline $\begin{array}{l}\text { Patients with VTE who } \\
\text { missed any dose of } \\
\text { VTE prophylaxis, } \mathrm{n} \\
(\%)\end{array}$ & $4 / 11(36.4)$ & $3 / 6(50.0)$ & $10 / 15(66.7)$ & 0.31 & \\
\hline \multicolumn{6}{|l|}{ Dose level } \\
\hline $\begin{array}{l}\text { Number of doses pre- } \\
\text { scribed }\end{array}$ & 5518 & 12,814 & 18,770 & & \\
\hline Missed doses, n (\%) & 215/5518 (3.9) & $1121 / 12,814$ (8.7) & $1494 / 18,770(8.0)$ & $<0.001$ & $0.82(0.77,0.87)^{*}$ \\
\hline Refused doses, n (\%) & $108 / 5518(2.0)$ & $735 / 12,814(5.7)$ & $982 / 18,770(5.2)$ & $<0.001$ & $0.76(0.71,0.82)^{*}$ \\
\hline $\begin{array}{l}\text { Other reasons for } \\
\text { missed doses, } \mathrm{n}(\%)\end{array}$ & $107 / 5,518(1.9)$ & $386 / 12,814(3.0)$ & $512 / 18,770(2.7)$ & $<0.001$ & $0.95(0.86,1.05)^{*}$ \\
\hline
\end{tabular}

$a O R$ adjusted odds ratio, $C I$ confidence interval, $D V T$ deep vein thrombosis, $P E$ pulmonary embolism, VTE venous thromboembolism

${ }^{\dagger} P$ values calculated using the Chi-square test and Fisher's exact test

$\S$ aOR calculated after adjusting for variables found to be statistically significantly different between the groups in Table 1 (age, race, ethnicity, hospital length of stay, body mass index, ventilation, and death)

"Separate univariate analysis, including only patients who were prescribed prophylaxis, showed a significant difference in race, ethnicity, hospital length of stay, body mass index, ventilation, and death between the three groups. Thus, in multiple logistic regression, we adjusted for these variables 
widespread recognition and amplified awareness of the high incidence and devastating consequences of VTE in patients with COVID-19.

While this study has some limitations (i.e., performed at a single academic center, lack of outpatient hospital-associated VTE events diagnosed after discharge), it is strengthened by a robust, validated methodology to identify missed doses of VTE prophylaxis $[6,10]$. Numerous interventions have been underway at JHH to improve administration of VTE prophylaxis [10], and our baseline rates are higher than other hospitals [6] which may also limit its generalizability to other hospitals.

We had hypothesized that decreased patient contact and approaches to conserving personal protective equipment might hinder administration of pharmacologic VTE prophylaxis in patients with COVID-19; however, this was not the case. Had our hypothesis been proven correct, then, successful educational interventions to prevent missed doses of VTE prophylaxis in hospitalized patients would have been a relatively easy solution to combat the high rates of VTE in patients with COVID-19 [10]. VTE events remain an important cause of mortality and morbidity in patients with COVID-19. These data should help allay fears that missed doses of pharmacologic VTE prophylaxis are contributing to VTE events in patients with COVID-19. Therefore, we should prioritize research to discover more effective approaches to VTE prevention in patients with COVID-19.

Authors Contribution VK contributed to project development, data management, data analysis, manuscript writing and editing. BDL contributed to project development, data management, and manuscript editing. OPO, PSK, and MBS, CGH, DBH, and DLS contributed to manuscript editing. ERH contributed to project development, manuscript writing and editing. All authors have read and approved the final version of the manuscript for submission.

Funding This research did not receive any specific grant from funding agencies in the public, commercial, or not-for-profit sectors.

Data availability Upon reasonable request.

\section{Compliance with ethical standards}

Conflict of interest Drs. Haut and Streiff, Ms. Holzmueller, Ms. Shaffer and Mr. Lau were supported by contracts from PCORI entitled "Preventing Venous Thromboembolism: Empowering Patients and Enabling Patient-Centered Care via Health Information Technology" (CE-12-11-4489) and "Preventing Venous Thromboembolism (VTE): Engaging Patients to Reduce Preventable Harm from Missed/Refused Doses of VTE Prophylaxis" (DI-1603-34596) and Implementing Best-Practice, Patient-Centered Venous Thromboembolism (VTE) Prevention in Trauma Centers" (DI-2019C3-17859). Mr. Lau, Ms. Varasteh Kia, Ms. Holzmueller, Ms. Shaffer, and Drs. Streiff, Haut, and Owodunni were/are supported by a grant from the AHRQ (1R01HS024547) entitled "Individualized Performance Feedback on Venous Thromboembolism Prevention Practice," a contract from PCORI entitled "Preventing Venous Thromboembolism (VTE): Engaging Patients to Reduce Preventable Harm from Missed/Refused Doses of VTE Prophylaxis," and a grant from the NIH/NHLBI (R21HL129028) entitled "Analysis of the Impact of Missed Doses of Venous Thromboembolism Prophylaxis." Drs. Haut, Streiff, Owodunni, Mr. Lau, and Ms. Varasteh Kia are supported by a grant from the AHRQ (R18HS025341) titled "Disseminating an Evidence-based Venous Thromboembolism Prevention Bundle." Mr. Lau is supported by the Institute for Excellence in Education Berkheimer Faculty Education Scholar Grant and a contract (AD-1306-03980) from PCORI entitled "Patient Centered Approaches to Collect Sexual Orientation/ Gender Identity Information in the Emergency Department." Dr. Haut is supported by a contract from the Patient-Centered Outcomes Research Institute (PCORI), "A Randomized Pragmatic Trial Comparing the Complications and Safety of Blood Clot Prevention Medicines Used in Orthopedic Trauma Patients" (PCS-1511-32745). Dr. Haut is a paid consultant and speaker for the "Preventing Avoidable Venous Thromboembolism-Every Patient, Every Time" VHA/Vizient IMPERATIV® Advantage Performance Improvement Collaborative. Dr. Haut receives royalties from Lippincott, Williams, Wilkins for a book-"Avoiding Common ICU Errors." Dr. Streiff has received research funding from Boehringer-Ingelheim, Janssen, Novo Nordisk, Roche and Sanofi and consulted for Bayer, Janssen, Pfizer and Portola and received honoraria for CME presentations from Bayer and Portola and has given expert witness testimony in various medical malpractice cases. Ms. Hobson and Dr. Kraus have nothing to disclose.

\section{References}

1. Goyal P, Choi JJ, Pinheiro LC et al (2020) Clinical characteristics of Covid-19 in Newyork city. N Engl J Med 382:2372-2374. https ://doi.org/10.1056/NEJMc2010419

2. Klok FA, Kruip MJHA, van der Meer NJM et al (2020) Confirmation of the high cumulative incidence of thrombotic complications in critically ill ICU patients with COVID-19: an updated analysis. Thromb Res 191:148-150. https://doi.org/10.1016/j.throm res.2020.04.041

3. COVID-19 and Coagulopathy-Hematology.org. https://www. hematology.org:443/covid-19/covid-19-and-coagulopathy. Accessed May 182020

4. Agency for healthcare research and quality, US department of health and human services. Preventing hospital-associated venous thromboembolism: a guide for effective quality improvement. https://www.ahrq.gov/sites/default/files/publications/files/vtegu ide.pdf. Accessed May 212020

5. Tang N, Bai H, Chen X et al (2020) Anticoagulant treatment is associated with decreased mortality in severe coronavirus disease 2019 patients with coagulopathy. J Thromb Haemost 18:10941099. https://doi.org/10.1111/jth.14817

6. Lau BD, Streiff MB, Kraus PS et al (2018) Missed doses of venous thromboembolism (VTE) prophylaxis at community hospitals: cause for alarm. J Gen Intern Med 33:19-20. https://doi. org/10.1007/s11606-017-4203-y

7. Shermock KM, Lau BD, Haut ER et al (2013) Patterns of nonadministration of ordered doses of venous thromboembolism prophylaxis: implications for novel intervention strategies. PLoS ONE 8:e66311. https://doi.org/10.1371/journal.pone.0066311

8. Haut ER, Lau BD, Kraus PS et al (2015) Preventability of hospital-acquired venous thromboembolism. JAMA Surg 150:912-915. https://doi.org/10.1001/jamasurg.2015.1340

9. Louis SG, Sato M, Geraci T et al (2014) Correlation of missed doses of enoxaparin with increased incidence of deep vein thrombosis in trauma and general surgery patients. JAMA Surg 149:365-370. https://doi.org/10.1001/jamasurg.2013.3963 
10. Haut ER, Aboagye JK, Shaffer DL et al (2018) Effect of real-time patient-centered education bundle on administration of venous thromboembolism prevention in hospitalized patients. JAMA Netw Open 1:e184741-e184741. https://doi.org/10.1001/jaman etworkopen.2018.4741
Publisher's Note Springer Nature remains neutral with regard to jurisdictional claims in published maps and institutional affiliations.

\section{Authors and Affiliations}

\section{Mujan Varasteh Kia ${ }^{1}$. Brandyn D. Lau ${ }^{2,3,4,11}$. Oluwafemi P. Owodunni ${ }^{1}$. Peggy S. Kraus ${ }^{9} \cdot$ Christine G. Holzmueller $^{4}$. Deborah B. Hobson ${ }^{1,4,10}$. Dauryne L. Shaffer ${ }^{1,10}$. Michael B. Streiff ${ }^{4,5}$. Elliott R. Haut ${ }^{1,4,6,7,8,11,12}$}

Brandyn D. Lau

blau2@jhmi.edu

Peggy S. Kraus

pkraus2@jhmi.edu

Deborah B. Hobson

dhobson1@jhmi.edu

1 Department of Surgery, Johns Hopkins University School of Medicine, Baltimore, MD, USA

2 Russell H. Morgan Department of Radiology and Radiological Science, Johns Hopkins University School of Medicine, Baltimore, MD, USA

3 Division of Health Sciences Informatics, Johns Hopkins University School of Medicine, Baltimore, MD, USA

4 The Armstrong Institute for Patient Safety and Quality, Johns Hopkins Medicine, Baltimore, MD, USA

5 Division of Hematology, Department of Medicine, Johns Hopkins University School of Medicine, Baltimore, MD, USA
6 Department of Anesthesiology and Critical Care Medicine, Johns Hopkins University School of Medicine, Baltimore, MD, USA

7 Department of Emergency Medicine, Johns Hopkins University School of Medicine, Baltimore, MD, USA

8 The Johns Hopkins Surgery Center for Outcomes Research, Johns Hopkins University School of Medicine, Baltimore, MD, USA

9 Department of Pharmacy, The Johns Hopkins Hospital, Baltimore, MD, USA

10 Department of Nursing, The Johns Hopkins Hospital, Baltimore, MD, USA

11 Department of Health Policy and Management, Johns Hopkins Bloomberg School of Public Health, Baltimore, MD, USA

12 Division of Acute Care Surgery, Department of Surgery, The Johns Hopkins University School of Medicine, Sheikh Zayed 6107C, 1800 Orleans St., Baltimore, MD 21287, USA 